\title{
ENTIRE FUNCTIONS THAT SHARE ONE VALUE WITH THEIR LINEAR DIFFERENTIAL POLYNOMIALS
}

\author{
PING LI*
}

\begin{abstract}
This paper study the problem on entire functions sharıng one value with their certain type of linear differential polynomials. The results here improved and generalized some results obtained by L. Z. Yang [9] and H. Zhong [10].
\end{abstract}

\section{Introduction}

Let $f$ and $g$ be two nonconstant meromorphic functions in the complex plane $\boldsymbol{C}$ and $a$ be a value in the extended complex plane $\overline{\boldsymbol{C}}$. We say that $f$ and $g$ share the value $a \mathrm{CM}$ (IM) provided that $f-a$ and $g-a(1 / f$ and $1 / g$, resp.) have the same zeros counting multiplicities (ignoring multiplicities) in the case of $a \in C$ $(a=\infty$, resp.). Rubel and Yang proved [8] that if an entire function $f$ share two finite values $\mathrm{CM}$ with its derivative, then $f \equiv f^{\prime}$. This result has been generalized to sharing values IM by G. Gundersen (see [3]) and by Mues-Steinmetz (see [7]) independently. In 1986, Jank, Mues and Volkmann [5] proved the following.

THEOREM A. Let $f(z)$ be a nonconstant entire function. If $f$ and $f^{\prime}$ share the value $a(a \neq 0) I M$, and $f^{\prime \prime}(z)=a$ when $f(z)=a$, then $f \equiv f^{\prime}$.

The function (see [10]) $f(z)=e^{a z}+a-1$, where $a \in C$ and $a \neq 1, a^{k-1}=1$, $k \geq 3$, shows that $f, f^{\prime}$ and $f^{(k)}$ share the value a CM, but $f \not \equiv f^{\prime}$ and $f \not \equiv f^{(k)}$. Hence the $f^{\prime \prime}$ in Theorem A can not be simply replaced by $f^{(k)}$ $(k \geq 3)$. In [10] Zhong gave a generalization of Theorem $\mathrm{A}$ in the following way.

THEOREM B. Let $f(z)$ be a nonconstant entire function. If $f$ and $f^{\prime}$ share the value $a(a \neq 0) C M$, and $f^{(n)}(z)=f^{(n+1)}(z)=a(n \geq 1)$ when $f(z)=a$, then $f \equiv f^{(n)}$.

1991 Mathematics Subject Classification Primary 30D35.

Key words and phrases. entıre function, sharing value, differential polynomial.

* The author was supported by the returned student fund of Academia Sinica.

Received November 5, 1998; revised April 26, 1999. 
Related to Zhong's result, L.-Z. Yang [9] gave another generalization of Theorem A.

THEOREM C. Let $f$ be a nonconstant entire function. If $f$ and $f^{(n)}$ share the finite value $a(a \neq 0) I M$, and $f^{\prime}(z)=f^{(n+1)}(z)=a$ when $f(z)=a$, then $f \equiv f^{(n)}$.

In this paper, we improve and generalize above results to the case that $f$ and its linear differential polynomials share one value. We prove the following theorems.

THEOREM 1. Let $f$ be a nonconstant entire function, and

$$
L=a_{1} f^{\prime}+a_{2} f^{\prime \prime}+\cdots+a_{n} f^{(n)},
$$

where $a_{1}, a_{2}, \ldots, a_{n} \neq 0$ are constants. If $f$ and $f^{\prime}$ share the finite and non-zero value a IM, and if $L(z)=L^{\prime}(z)=a$ when $f(z)=a$, then $f=f^{\prime}=L$.

THEOREM 2. Let $f$ be a nonconstant entire function, and $L$ be the differential polynomial in $f$ defined in (1) whose coefficients satisfy $\sum_{k=1}^{n} 2^{k} a_{k} \neq 0$ or $\sum_{k=1}^{n} a_{k} \neq-1$. If $f$ and $L$ share the finite and non-zero value $a I M$, and if $f^{\prime}(z)=L^{\prime}(z)=a$ when $f(z)=a$, then $f=f^{\prime}=L$.

We will give an example to show that the condition $\sum_{k=1}^{n} 2^{k} a_{k} \neq 0$ or $\sum_{k=1}^{n} a_{k} \neq-1$ in Theorem 2 is necessary.

We assume that the reader is familiar with the basic notations and results about Nevanlinna's value distribution theory (see [4] or [6]).

\section{Lemmas}

LEMMA 1 (see [10]). Let $f$ be a nonconstant entire function. If $f$ and $f^{\prime}$ share the finite and non-zero value a IM, then

$$
T(r, f) \leq 2 N\left(r, \frac{1}{f-a}\right)+S(r, f),
$$

where and in the sequel the notation $S(r, f)$ is defined to be any quantity satisfying $S(r, f)=o(T(r, f))$, as $r \rightarrow \infty$ possibly outside a set of finite linear measure.

LEMMA 2 (Clunie [1], Doeringer [2]). Let $f$ be a nonconstant meromorphic function and $Q[f], Q^{*}[f]$ be differential polynomials in $f$ with $Q[f] \not \equiv 0$. Let $n \in N$ and

$$
f^{n} Q^{*}[f]=Q[f]
$$

If the degree of $Q[f]$ is not great than $n$, then $m\left(r, Q^{*}[f]\right)=S(r, f)$. 
Lemma 3. Let $\varphi(\not \equiv 0)$ be an entire function. If $\varphi^{n}+P[\varphi] \equiv 0$, where $P[\varphi]$ is a differential polynomial in $\varphi$ with constant coefficients, and the degree of $P[\varphi]$ is at most $n-1$, then $\varphi$ is a constant.

Proof. Suppose $\varphi$ is not a constant. We write $\varphi^{n}+P[\varphi] \equiv 0$ as $\varphi^{n-1} \cdot \varphi=$ $-P[\varphi]$. Then by Lemma 2, we have $m(r, \varphi)=S(r, \varphi)$. Note that $\varphi$ is an entire function. We get $T(r, \varphi)=S(r, \varphi)$, a contradiction. Hence $\varphi$ is a constant.

LEMMA 4. Let $f$ be a meromorphic function, $L$ be the differential polynomial in $f$ defined in (1), and $a$ be a finite non-zero value. Then

$$
m\left(r, \frac{1}{f-a}\right)+m\left(r, \frac{1}{L-a}\right) \leq m\left(r, \frac{1}{L^{\prime}}\right)+S(r, f) .
$$

Proof. By the lemma of logarithmic derivative, we have $m(r, L /(f-a))=$ $S(r, f)$ and $m\left(r, L^{\prime} /(L(L-a))\right)=S(r, f)$. In terms of Nevanlinna first fundamental theorem and the properties of the counting functions, we have

$$
\begin{aligned}
m\left(r, \frac{1}{L}\right)+m\left(r, \frac{1}{L-a}\right) & =2 T(r, L)-N\left(r, \frac{1}{L}\right)-N\left(r, \frac{1}{L-a}\right)+O(1) \\
& =T\left(r, \frac{1}{L(L-a)}\right)-N\left(r, \frac{1}{L(L-a)}\right)+O(1) \\
& =m\left(r, \frac{1}{L(L-a)}\right)+O(1)
\end{aligned}
$$

Hence

$$
\begin{aligned}
m\left(r, \frac{1}{f-a}\right)+m\left(r, \frac{1}{L-a}\right) & =m\left(r, \frac{L}{f-a} \cdot \frac{1}{L}\right)+m\left(r, \frac{1}{L-a}\right) \\
& \leq m\left(r, \frac{L}{f-a}\right)+m\left(r, \frac{1}{L}\right)+m\left(r, \frac{1}{L-a}\right)+S(r, f) \\
& \leq m\left(r, \frac{1}{L(L-a)}\right)+S(r, f) \\
& \leq m\left(r, \frac{L^{\prime}}{L(L-a)}\right)+m\left(r, \frac{1}{L^{\prime}}\right)+S(r, f) \\
& \leq m\left(r, \frac{1}{L^{\prime}}\right)+S(r, f)
\end{aligned}
$$

LEMMA 5. Let $f$ be a nonconstant entire function, $L$ be the differential polynomial in $f$ defined in (1), and $f \not \equiv L$. If $f$ and $L$ share the finite and non-zero 
value a IM, and if $f^{\prime}(z)=L^{\prime}(z)=a$ when $f(z)=a$, then

$$
T(r, f)=2 N\left(r, \frac{1}{f-a}\right)+S(r, f) .
$$

Proof. Since $f^{\prime}(z)=L(z)=L^{\prime}(z)=a$ when $f(z)=a$ and $f \not \equiv L$, we can derive that

$$
\begin{aligned}
N\left(r, \frac{1}{f-a}\right) & \leq \frac{1}{2} N\left(r, \frac{1}{f-L}\right)+S(r, f) \\
& \leq \frac{1}{2} T(r, f-L)+S(r, f) \\
& \leq \frac{1}{2} m(r, f-L)+S(r, f) \\
& \leq \frac{1}{2} m(r, f)+\frac{1}{2} m\left(r, 1-\frac{L}{f}\right)+S(r, f) \\
& \leq \frac{1}{2} m(r, f)+S(r, f) \\
& \leq \frac{1}{2} T(r, f)+S(r, f) .
\end{aligned}
$$

On the other hand, by Lemma 4 , we have

$$
\begin{aligned}
T(r, f)+T(r, L) & \leq N\left(r, \frac{1}{f-a}\right)+N\left(r, \frac{1}{L-a}\right)+m\left(r, \frac{1}{L^{\prime}}\right)+S(r, f) \\
& \leq 2 N\left(r, \frac{1}{f-a}\right)+T\left(r, L^{\prime}\right)+S(r, f)
\end{aligned}
$$

which leads to

$$
T(r, f) \leq 2 N\left(r, \frac{1}{f-a}\right)+S(r, f) .
$$

Hence $T(r, f)=2 N(r, 1 /(f-a))+S(r, f)$.

LEMMA 6. Let $f$ be a nonconstant entire function, $L$ be the differential polynomial in $f$ defined in (1), and $a$ be a finite non-zero value satisfying $m(r, 1 /(f-a))=S(r, f)$. If $f^{\prime}(z)=L(z)=L^{\prime}(z)=a$ when $f(z)=a$, then $f=$ $f^{\prime}=L$.

\section{Proof. Set}

$$
\alpha=\frac{f^{\prime}-a}{f-a} .
$$

Since $a$ is a non-zero value and $f^{\prime}(z)=a$ when $f(z)=a$, all $a$-points of $f$ are simple, and thus $\alpha$ is an entire function. By the lemma of logarithmic derivative and the condition $m(r, 1 /(f-a))=S(r, f)$, we deduce that $m(r, \alpha)=S(r, f)$. 
Hence

$$
T(r, \alpha)=S(r, f) .
$$

The equation (2) can be rewritten as

$$
f^{\prime}=a+\alpha(f-a)=\beta_{1}+\alpha_{1} f
$$

where $\beta_{1}=a-a \alpha$ and $\alpha_{1}=\alpha$ are entire functions. From (4), we deduce that

$$
f^{(k)}=\beta_{k}+\alpha_{k} f
$$

where $\beta_{k}$ and $\alpha_{k}$ are entire functions satisfying the following recurrence formulas

$$
\begin{aligned}
& \beta_{k+1}=\beta_{k}^{\prime}+\beta_{1} \alpha_{k}, \\
& \alpha_{k+1}=\alpha_{k}^{\prime}+\alpha_{1} \alpha_{k},
\end{aligned}
$$

for $k=1,2, \ldots$ Hence we have

$$
L=\sum_{k=1}^{n} a_{k} f^{(k)}=\xi+\eta f,
$$

where

$$
\xi=\sum_{k=1}^{n} a_{k} \beta_{k} \quad \text { and } \quad \eta=\sum_{k=1}^{n} a_{k} \alpha_{k} .
$$

From the recurrence formulas (6) and (7), we can easily see that $\xi$ and $\eta$ are entire functions, and

$$
T(r, \xi)=S(r, f), \quad T(r, \eta)=S(r, f) .
$$

By taking the derivative in equation (8), we get

$$
L^{\prime}=\xi^{\prime}+\eta^{\prime} f+\eta f^{\prime} .
$$

Suppose $z$ is an $a$-point of $f$. Then by the assumption of Lemma 6 and equations (8), (10), we have

$$
\begin{aligned}
& \xi(z)+\eta(z) a-a=0, \\
& \xi^{\prime}(z)+\eta^{\prime}(z) a+\eta(z) a-a=0 .
\end{aligned}
$$

Let $\gamma_{1}=\xi+\eta a-a$ and $\gamma_{2}=\xi^{\prime}+\eta^{\prime} a+\eta a-a$. We have $\gamma_{1}(z)=0, \gamma_{2}(z)=0$, and $T\left(r, \gamma_{1}\right)=S(r, f), T\left(r, \gamma_{2}\right)=S(r, f)$. If $\gamma_{1} \not \equiv 0$ or $\gamma_{2} \not \equiv 0$, say, $\gamma_{1} \not \equiv 0$, then we get

$$
N\left(r, \frac{1}{f-a}\right) \leq N\left(r, \frac{1}{\gamma_{1}}\right) \leq T\left(r, \gamma_{1}\right)+S(r, f)=S(r, f) .
$$

This and the condition $m(r, 1 /(f-a))=S(r, f)$ lead to $T(r, f)=S(r, f)$, a contradiction. Hence we have $\gamma_{1} \equiv 0$ and $\gamma_{2} \equiv 0$, which imply $\xi \equiv 0$ and $\eta \equiv 1$ 
or

$$
\sum_{k=1}^{n} a_{k} \alpha_{k} \equiv 1
$$

Hence from (8) we get $L=f$. By recurrence formula (7), equation (13) can be expressed as

$$
a_{n} \alpha^{n}+P[\alpha] \equiv 0,
$$

where $P[\alpha]$ is a differential polynomial in $\alpha$ with degree less than or equal to $n-1$. From this and by using Lemma 3, we can conclude that $\alpha$ is a constant. Therefore from recurrence formula (7), we get $\alpha_{k}=\alpha^{k}$. From (4), we deduce that

$$
\begin{aligned}
f & =-\frac{a(1-\alpha)}{\alpha}+A e^{\alpha z}, \\
f^{(k)} & =\alpha^{k} A e^{\alpha z}=\alpha_{k} A e^{\alpha z}, \quad k=1,2, \ldots, n,
\end{aligned}
$$

where $A$ is a non-zero constant. Note $\sum_{k=1}^{n} a_{k} \alpha_{k}=1$. From the above equation, we have

$$
L=\left(\sum_{k=1}^{n} a_{k} \alpha_{k}\right) A e^{\alpha z}=A e^{\alpha z}=\frac{1}{\alpha} f^{\prime} .
$$

Let $z$ be an $a$-point of $f$. We get $\alpha=f^{\prime}(z) / L(z)=1$, and thus $L=f^{\prime}$. Hence $f=f^{\prime}=L$, which completes the proof of Lemma 6 .

LEMMA 7. Let $f$ be a nonconstant entire function, $L$ be the differential polynomial in $f$ defined in (1), and $a$ be a finite non-zero value sattsfying $N(r, 1 /(f-a)) \neq S(r, f)$. If $f^{\prime}(z)=L(z)=L^{\prime}(z)=a$ when $f(z)=a$, then $f^{\prime} \not \equiv$ $L-f+a$.

\section{Proof. Suppose}

$$
f^{\prime} \equiv L-f+a
$$

Then we have $f^{\prime \prime} \equiv L^{\prime}-f^{\prime}$. Therefore according to the assumption we have $f^{\prime \prime}(z)=0$ when $f(z)=a$. Set

$$
h=\frac{f^{\prime \prime}}{f-a} .
$$

We see that $h$ is an entire function and $T(r, h)=S(r, f)$. Rewrite $(15)$ as $f^{\prime \prime}=$ $h(f-a)$ and taking the derivatives, we can get

$$
f^{(k+2)}=\lambda_{k}(f-a)+\mu_{k} f^{\prime}, \quad k=0,1, \ldots,
$$


where $\lambda_{0}=h, \mu_{0}=0$, and $\lambda_{k}, \mu_{k}$ are entire functions satisfying the following recurrence formulas

$$
\begin{aligned}
& \lambda_{k}=\lambda_{k-1}^{\prime}+h \mu_{k-1}, \\
& \mu_{k}=\lambda_{k-1}+\mu_{k-1}^{\prime},
\end{aligned}
$$

for $k=1,2, \ldots$ Hence by (16) we have

$$
\begin{aligned}
L & =a_{1} f^{\prime}+\sum_{k=2}^{n} a_{k} f^{(k)} \\
& =\left(\sum_{k=2}^{n} a_{k} \lambda_{k-2}\right)(f-a)+\left(a_{1}+\sum_{k=2}^{n} a_{k} \mu_{k-2}\right) f^{\prime} .
\end{aligned}
$$

Note that $f^{\prime}(z)=L(z)=a$ when $f(z)=a$. We have $a_{1}+\sum_{k=2}^{n} a_{k} \mu_{k-2}(z)=1$ for all $a$-points of $f$. From the recurrence formulas (17) and (18), we see that $T\left(r, \lambda_{k}\right)=S(r, f), T\left(r, \mu_{k}\right)=S(r, f), k=1,2, \ldots \quad$ Since $N(r, 1 /(f-a)) \neq$ $S(r, f)$, we can conclude that

$$
a_{1}+\sum_{k=2}^{n} a_{k} \mu_{k-2} \equiv 1,
$$

and thus by (19) and (14), we have

$$
\sum_{k=2}^{n} a_{k} \lambda_{k-2} \equiv 1 .
$$

From the recurrence formulas (17) and (18), by mathematical induction in the number $k$, we can conclude that $\lambda_{k}$ and $\mu_{k+1}$ are differential polynomials in $h$ with degree less than or equal to $(k+1) / 2$ when $k$ is odd; and $\lambda_{k}$ and $\mu_{k+1}$ have the forms $h^{(k+2) / 2}+P_{k}[h]$ and $h^{(k+2) / 2}+Q_{k}[h]$, respectively, when $k$ is even, where $P_{k}[h]$ and $Q_{k}[h]$ are differential polynomials in $h$ with degree less than or equal to $k / 2$. Hence for even number $n \geq 2$ equation (21) can be expressed as

$$
h^{n / 2}+P[h] \equiv 0
$$

and for odd number $n \geq 3$ equation (20) can be expressed as

$$
h^{(n-1) / 2}+Q[h] \equiv 0,
$$

where $P[h]$ and $Q[h]$ are differential polynomials in $h$ with $\operatorname{deg} P[h] \leq(n / 2)-1$ and $\operatorname{deg} Q[h] \leq((n-1) / 2)-1$. In both cases, by using Lemma 3 , we can conclude that $h$ is a constant. Hence by $(15)$, we get $\left(f^{\prime}\right)^{2}=h(f-a)^{2}+C_{1}$, where $C_{1}$ is a constant. By assumptions of Lemma 7, the constant $C_{1}$ should be $a^{2}$. Therefore we have

$$
\left(f^{\prime}-a\right)\left(f^{\prime}+a\right)=h(f-a)^{2} .
$$


By assumptions of Lemma 7 again, we see that $-a$ is an exceptional value of $f^{\prime}$. Hence there exists an entire function $\zeta$ such that $f^{\prime}=-a+e^{\zeta}$, and thus $f^{\prime \prime}=\zeta^{\prime} e^{\zeta}$. Combining this and (15), we see that $\zeta^{\prime}(z)=0$ when $f(z)=a$. Note that $N(r, 1 /(f-a)) \neq S(r, f)$ and $T(r, \zeta)=S(r, f)$. If $\zeta^{\prime} \not \equiv 0$, then we get $N(r, 1 /(f-a)) \leq N\left(r, 1 / \zeta^{\prime}\right)=S(r, f)$, a contradiction. Therefore we have $\zeta^{\prime} \equiv$ 0 . Hence we see that $f^{\prime}$ is a constant, and thus by (24) $f$ is a constant, which contradicts the assumption and completes the proof of Lemma 7.

\section{The proofs of theorems}

Proof of Theorem 1. Set

$$
\phi=\frac{L^{\prime}-f^{\prime}}{f-a}, \quad \phi_{1}=\frac{L-f^{\prime}}{f-a} .
$$

It is easily seen that $\phi, \phi_{1}$ are entire functions and $T(r, \phi)=S(r, f), T\left(r, \phi_{1}\right)=$ $S(r, f)$.

If $\phi \not \equiv 0$, then we have

$$
m(r, f)=m\left(r, a+\frac{1}{\phi}\left(L^{\prime}-f^{\prime}\right)\right) \leq m\left(r, f^{\prime}\right)+S(r, f) \leq m(r, f)+S(r, f),
$$

and thus $T(r, f)=T\left(r, f^{\prime}\right)+S(r, f)$. From (25), we have $f=a+(1 / \phi)$. $\left(L^{\prime}-f^{\prime}\right)$. By taking the derivative, we get

$$
f^{\prime}=\left(\frac{1}{\phi}\right)^{\prime}\left(L^{\prime}-f^{\prime}\right)+\frac{1}{\phi}\left(L^{\prime \prime}-f^{\prime \prime}\right)
$$

which leads to

$$
\left(1+\left(\frac{1}{\phi}\right)^{\prime}\right) f^{\prime}=\left(\frac{1}{\phi}\right)^{\prime} L^{\prime}+\frac{1}{\phi}\left(L^{\prime \prime}-f^{\prime \prime}\right)
$$

Since $\phi$ is an entire function, we can easily see that $1+(1 / \phi)^{\prime} \not \equiv 0$. Therefore

$$
\frac{f^{\prime}}{f^{\prime}-a}=\frac{1}{1+\left(\frac{1}{\phi}\right)^{\prime}}\left\{\left(\frac{1}{\phi}\right)^{\prime} \frac{L^{\prime}}{f^{\prime}-a}+\frac{1}{\phi} \frac{L^{\prime \prime}-f^{\prime \prime}}{f^{\prime}-a}\right\} \text {. }
$$

Hence from the above equation and by using the lemma of logarithmic derivative, we can deduce that $m\left(r, f^{\prime} /\left(f^{\prime}-a\right)\right)=S(r, f)$, and thus

$$
m\left(r, \frac{1}{f^{\prime}-a}\right)=m\left(r, \frac{f^{\prime}}{f^{\prime}-a}\right)+O(1)=S(r, f) .
$$

Since $L^{\prime}=\sum_{k=1}^{n} a_{k} f^{(k+1)}$ and $L^{\prime}(z)=a$ when $f^{\prime}(z)=a$, we see that the multiplicity of any $a$-point of $f^{\prime}$ is at most $n$. Hence we have 


$$
N_{(2}\left(r, \frac{1}{f^{\prime}-a}\right) \leq n \bar{N}_{(2}\left(r, \frac{1}{f^{\prime}-a}\right) \text {, }
$$

where $N_{(2}\left(r, 1 /\left(f^{\prime}-a\right)\right)$ and $\bar{N}_{(2}\left(r, 1 /\left(f^{\prime}-a\right)\right)$ are defined to be the counting function and reduced counting function of $1 /\left(f^{\prime}-a\right)$ related to the multiple $a$-points of $f^{\prime}$, respectively. Suppose $z$ is a multiple $a$-point of $f^{\prime}$. Then by calculation we have $\phi_{1}(z)=1$. By Lemma 1 , we have $N(r, 1 /(f-a)) \neq S(r, f)$. Therefore from Lemma 7 , we see that $\phi_{1} \not \equiv 1$. Hence $\bar{N}_{(2}\left(r, 1 /\left(f^{\prime}-a\right)\right) \leq$ $N\left(r, 1 /\left(\phi_{1}-1\right)\right) \leq S(r, f)$, and thus $N_{(2}\left(r, 1 /\left(f^{\prime}-a\right)\right) \leq S(r, f)$, which implies that

$$
N\left(r, \frac{1}{f^{\prime}-a}\right)=\bar{N}\left(r, \frac{1}{f^{\prime}-a}\right)+S(r, f)
$$

Therefore

$$
\begin{aligned}
m\left(r, \frac{1}{f-a}\right) & =T(r, f)-N\left(r, \frac{1}{f-a}\right)+S(r, f) \\
& =T\left(r, f^{\prime}\right)-N\left(r, \frac{1}{f-a}\right)+S(r, f) \\
& =N\left(r, \frac{1}{f^{\prime}-a}\right)-N\left(r, \frac{1}{f-a}\right)+S(r, f) \\
& =\bar{N}\left(r, \frac{1}{f^{\prime}-a}\right)-N\left(r, \frac{1}{f-a}\right)+S(r, f) \\
& =S(r, f) .
\end{aligned}
$$

Hence by Lemma 6 , we obtain $f=f^{\prime}=L$, which leads to $\phi \equiv 0$, a contradiction.

If $\phi \equiv 0$, then $L-f$ is a constant. By Lemma 1 , we see that the value $a$ is not an exceptional value of $f$. Hence we must have $L-f=0$. From this we can get

$$
m\left(r, \frac{1}{f-a}\right)=m\left(r, \frac{f}{f-a}\right)+O(1)=m\left(r, \frac{L}{f-a}\right)+O(1)=S(r, f),
$$

and thus by Lemma 6 , we conclude that $f=f^{\prime}=L$, which completes the proof of Theorem 1.

Proof of Theorem 2. Let $\phi$ be the function defined in (25) and

$$
\psi=\frac{L^{\prime}}{f-a}-\frac{L^{\prime}}{L-a}=\frac{L^{\prime}(L-f)}{(f-a)(L-a)} .
$$

It is obvious that $m(r, \phi)=S(r, f)$ and $m(r, \psi)=S(r, f)$. Since $a \neq 0$ and $f(z)=a$ implies $f^{\prime}(z)=L(z)=L^{\prime}(z)=a$, we see that all $a$-points of $f$ are simple 
and these points are double zeros of $L-f$. Therefore $\phi$ and $\psi$ are entire functions. Hence

$$
T(r, \phi)=S(r, f), \quad T(r, \psi)=S(r, f) .
$$

Suppose $z$ is an $a$-point of $f$. Then by calculation, we have

$$
\phi(z)=\frac{L^{\prime \prime}(z)-f^{\prime \prime}(z)}{a}, \quad \psi(z)=\frac{1}{2} \frac{L^{\prime \prime}(z)-f^{\prime \prime}(z)}{a},
$$

and thus $\phi(z)-2 \psi(z)=0$. If $\phi-2 \psi \not \equiv 0$, then we obtain $N(r, 1 /(f-a))=$ $S(r, f)$. From this and Lemma 5, we get $T(r, f)=S(r, f)$, a contradiction. Hence we have $\phi \equiv 2 \psi$, which leads to $L-f=c(L-a)^{2}$ or

$$
f-a=(L-a)(1+c a-c L),
$$

where $c$ is a constant. If $c=0$, then we have $f=L$, and thus

$$
m\left(r, \frac{1}{f-a}\right)=m\left(r, \frac{f}{f-a}\right)+S(r, f)=m\left(r, \frac{L}{f-a}\right)+S(r, f)=S(r, f) .
$$

Hence by Lemma 6 , we obtain the conclusion $f=f^{\prime}=L$.

Suppose now that $c \neq 0$. If $a+(1 / c)$ is not an exceptional value of $L$, then there exists a $z$ such that

$$
1+c a-c L(z)=0 .
$$

Therefore from equation (27), we have $f(z)-a=0$, and thus by assumption $L(z)=a$, which contradicts the above equation. Hence $a+(1 / c)$ is an exceptional value of $L$. This means that there exists an entire function $\gamma$ such that

$$
L=a+\frac{1}{c}+e^{\gamma}
$$

Obviously, we have $T(r, \gamma) \leq S(r, L) \leq S(r, f)$.

If $a+(1 / c) \neq 0$, then we have

$$
m\left(r, \frac{1}{L}\right)=m\left(r, \frac{1}{e^{\gamma}+a+\frac{1}{c}}\right) \leq S\left(r, e^{\gamma}\right) \leq S(r, f) .
$$

From this and by using Lemma 5 , we have

$$
T(r, f)=2 m\left(r, \frac{1}{f-a}\right)+S(r, f) \leq 2 m\left(r, \frac{1}{L}\right)+S(r, f) \leq S(r, f),
$$

a contradiction. Hence $a+(1 / c)=0$. This, (27) and (28) give

$$
f=a+\frac{1}{a} L(L-a), \quad L=e^{\gamma}
$$


and thus $L^{\prime}=\gamma^{\prime} L$. Note that $N(r, 1 /(f-a)) \neq S(r, f)$ and $f(z)=a$ implies $L(z)=L^{\prime}(z)=a$. We must have $\gamma^{\prime} \equiv 1$ or $L^{\prime}=L$. From the above equation, we get $f^{(k)}=\left(2^{k} / a\right) L^{2}-L, k=1,2, \ldots, n$. Therefore

$$
L=\sum_{k=1}^{n} a_{k} f^{(k)}=\left(\sum_{k=1}^{n} 2^{k} a_{k}\right) \frac{1}{a} L^{2}-\left(\sum_{k=1}^{n} a_{k}\right) L .
$$

Note that $L$ is not a constant. Hence we have

$$
\sum_{k=1}^{n} 2^{k} a_{k}=0, \quad \sum_{k=1}^{n} a_{k}=-1
$$

which contradict the assumption, and completes the proof of Theorem 2 .

The following example shows that the condition $\sum_{k=1}^{n} 2^{k} a_{k} \neq 0$ or $\sum_{k=1}^{n} a_{k} \neq-1$ in Theorem 2 in necessary.

EXAMPLE 1. Suppose $a_{1}, a_{2}, \ldots, a_{n}$ are constants satisfying

$$
\sum_{k=1}^{n} 2^{k} a_{k}=0, \quad \sum_{k=1}^{n} a_{k}=-1
$$

Let $f(z)=a+(1 / a) e^{2 z}-e^{z}$, where $a$ is a finite non-zero constant. Then $f^{(k)}(z)=\left(2^{k} / a\right) e^{2 z}-e^{z}$, and $L(z):=\sum_{k=1}^{n} a_{k} f^{(k)}(z)=e^{z}$. Hence $f, L$ share $a$ $\mathrm{CM}$, and $f^{\prime}(z)=L(z)=L^{\prime}(z)=a$ when $f(z)=a$, but $f \neq f^{\prime}$.

Acknowledgement. The author wants to thank the referee for his/her thorough reviewing with useful comments made to the paper.

\section{REFERENCES}

[ 1 ] J. Clunie, On integral and meromorphic functions, J. London Math. Soc., 37 (1962), 17-27.

[2] W. DoERINGER, Exceptıonal values of differentıal polynomial, Pacific J. Math., 98 (1982), 5562.

[3] G. G. GunderSEN, Meromorphic functions that share finite values with their derivative, J. Math. Anal. Appl., 75 (1980), 441-446.

[4] W K. Hayman, Meromorphic Functions, Clarendon Press, Oxford, 1964.

[5] G. Jank, E. Mues AND L. VolkmanN, Meromorphe funktionen, die mit iher ersten und Zweiten Ableitung einen endlichen Wert teilen, Complex Variables Theory Appl., 6 (1986), 51-71.

[6] I. LaINE, Nevanlinna Theory and Complex Differentıal Equations, Water de Gruyter, New York, 1993.

[7] E. Mues and N. Steinmetz, Meromorphe Funktionen, die mit ihrer Ableitung Werte teilen, Manuscripta Math., 29 (1979), 195-206.

[ 8 ] L. A. Rubel AND C. C. YANG, Values shared by an entıre function and its derivative, Lecture Notes in Math., 599, Springer-Verlag, Berlin, 1977, 101-103. 
[9] L. Z. YANG, Entıre functions that share one value with their derivatives, Bull. Hong Kong Math. Soc., 2 (1998), 115-121.

[10] H. Zhong, Entıre functions that share one value with their derivatives, Kodai Math. J., 18 (1995), 250-259.

Department OF MATHEMATiCS

The University of Science and Technology of China

Hefei ANHuI, 230026, P.R. China

E-mail: pli@ustc.edu.cn 